\title{
FIRST X-RAY EVIDENCE OF HETEROGENEOUS IMPURITY CORRELATIONS IN VERY HIGHLY DOPED $n$-GaAs
}

\author{
T. SLUPIŃSKI* AND E. ZIELIŃSKA-ROHOZIŃSKA \\ Institute of Experimental Physics, Warsaw University \\ Hoża 69, 00-681 Warszawa, Poland
}

\begin{abstract}
Measurements of X-ray scattering from very highly doped GaAs:Te single crystals as a function of doping level and thermal treatment (annealing temperature) are reported. Reversible diffuse X-ray scattering occurs after sample annealing below a certain temperature. Presented results indicate an inhomogeneous arising of impurity-impurity correlations in GaAs:Te solid solution. Observed features of diffuse $\mathrm{X}$-ray scattering in reciprocal space can be well understood within Krivoglaz theory of scattering due to spatial fluctuations of solute atoms pair correlation function and related lattice deformations. Good coincidence of diffuse X-ray scattering with the free electron concentration changes caused by an annealing is reported. Free electron concentration drop accompanying impurity correlation strongly suggests a certain form of impurity bonding.
\end{abstract}

PACS numbers: 61.72.-y, 61.10.-i, 71.55.-i

Highly doped semiconductors are routinely approximated by ideal solid solutions. Many experimental phenomena not consistent with such a model are accounted by introducing point defects (e.g. vacancies) and treating a semiconductor as double ideal solution. Here we briefly present X-ray scattering arguments that highly doped GaAs:Te exhibits properties of nonideal solid solution, i.e. one with spatial correlations of solute atoms.

In the investigations of critical phenomena the scattering experiments supply the fundamental information on microscopic correlations, order parameters and fluctuations [1, 2]. Measurements of the critical scaltering [3], with its intensity dependence on temperature of the type $I \propto\left(T_{\mathrm{c}}-T\right)^{x}, T_{\mathrm{c}}$ is a critical temperature and $x$ a certain index, allowed to derive many well-known conclusions on phase transitions $[1,2]$. The best known are the density fluctuations which occur close to gas-liquid critical point, detected by light scattering (critical opalescence). Another one is neutron scattering detection of the order of atomic magnetic moments, which occurs in a ferromagnet when Curie temperature is approached from above.

\footnotetext{
*e-mail: tomslu@fuw.edu.pl.
} 
An important example from our point is the scattering of neutrons from $\beta$-brass (Zn-Cu 1:1 alloy) which undergoes order-disorder transition at $T_{\mathrm{c}}$ [4] or X-ray scattering from $\mathrm{Cu}_{3} \mathrm{Au}$ alloy with a similar transition, where short-range order parameters close to $T_{\mathrm{c}}$ were measured ([5], Ch. 12 of Ref. [6]).

It is proved (e.g. see Ch. $2 \mathrm{C}$ of Ref. [4] or pp. 806-8 of Ref. [2]) that the scattering intensity in the first Born approximation is, in principal, a direct measure of the Fourier components of the pair (or two-point) correlation function (static) defined by

$$
\tilde{g}\left(\boldsymbol{r}_{i}, \boldsymbol{r}_{j}\right)=\left\langle\left[s\left(r_{i}\right)-\left\langle s\left(r_{i}\right)\right\rangle\right]\left[s\left(r_{j}\right)-\left\langle s\left(r_{j}\right)\right\rangle\right]\right\rangle,
$$

where $s\left(r_{i}\right)$ denotes a certain atomic parameter causing the scattering, located at lattice site $r_{i}$ (e.g. spin in magnetic systems or lattice point occupation with solute atom in case of alloys). Brackets ( ) denote an averaging over statistical ensemble. Such pair correlation function in case of an alloy crystal is a normalised measure of the fact that solute atoms occupy simultaneously sites $i$ and $j$. Local correlation function $g\left(r_{i}, \rho\right)=\tilde{g}\left(r_{i}, r_{i}+\rho\right)$ for a given $\rho=r_{j}-r_{i}-$ a distance between lattice sites $i$ and $j$, is a function of a coordinate $r_{i}$ and among others, in an inhomogeneous crystal it contains an information on the heterogeneities fluctuations of short-range order parameter in a solid solution.

While neutron scattering was mainly used in alloys to observe an order at crystal lattice sites (not static displacement of atoms from their sites in an ideal lattice), modern high resolution X-ray diffraction techniques (HR-XRD) in very high quality single crystals allow to recognise extended static lattice deformations, which can arise as an effect of two-point correlations. The theoretical basis was created many years ago by Krivoglaz [8]. He applied the harmonic approximation to a crystal lattice of a solid solution and showed that the Fourier components of the lattice deformation field $u_{k}$ and of the solute atoms pair correlation function $g_{k}(\rho)$ are related

$$
u_{k}=\sum_{\rho} A_{k}(\rho) g_{k}(\rho),
$$

where $k$ is a wave vector, $\boldsymbol{A}_{k}(\boldsymbol{\rho})$ is a vector proportional to the inverse dynamical matrix (see e.g. page 16 in Ref. [9]) of the crystal lattice and to a vector of force constants between solute atoms at sites $i$ and $j$.

As it was shown by many authors, if static ([10,8] page 130 in Ref. [11]) or dynamic (ch. 11 in Ref. [6]) deviations from an ideal periodicity in a crystal exist (atoms are displaced at $u\left(r_{i}\right)$ from their sites in an ideal lattice) then in the first Born approximation (kinematical theory of scattering) the diffuse X-ray scattering (DXS) arises, additionally to Bragg diffraction from an average crystal lattice. For a case of static deviations DXS is given by [11] (when all sublattices are deformed without a phase shift between them - analogous to an acoustic mode of lattice oscillations):

$$
I_{\mathrm{diff}}\left(h_{h k l}+q\right) \propto\left|F_{h k l}\right|^{2} \cdot\left|\left(h_{h k l}+q\right) \cdot u_{q}\right|^{2},
$$

where $F_{h k l}$ is a geometrical structure factor, $u_{q}$ is $q$ Fourier component of deformation field, $h_{h k l}$ is a vector of reciprocal lattice point and $h_{h k l}+q$ is equal to the scattering vector $\Delta k$. An occurrence of scattered intensity for a vector 
$\Delta k=h_{h k l}+q$ near the Bragg reflection $\Delta k=h_{h k l}$ indicates an existence of $q$ component of lattice deformation field. Thus from the above it is seen that the fluctuations of pair correlation function with a length $2 \pi / q$ will cause diffuse scattering for wave vector $q$ around Bragg reflections. An approximate relation for $|q| \ll|h|$ can be derived from above Krivoglaz results

$$
I_{\text {diff }}(q) \propto f(q / q)\left|g_{q}\right|^{2} / q^{4}
$$

for a case of one type of correlations in a crystal of nonideal solid solution, where $f(q / q)$ is an angular factor. Krivoglaz concluded $[8,11]$ that the origin of lattice deformations in a solid solution can be recognised by an analysis of scattered intensity profiles in the reciprocal space (angular dependence). The lattice deformations coming from the fluctuations of solute atom-atom correlations produce the isointensity surfaces with a torus-like shape around the diffraction vector $h$ (or oval in a plane), and one coming from fluctuations of solute concentration gives isointensity surfaces of a shape of two spheres aligned along $h$.

Below we present the results of X-ray scattering from GaAs:Te as a function of doping level and sample thermal treatment, which correspond well to the scattering description sketched above. It was already reported that an annealing causes reversible changes of the free electron concentration in sufficiently highly doped $n$-GaAs $[12,13]$. Here similarly reversible changes of DXS are reported.

The samples of GaAs:Te were cut from single crystals of high quality (X-ray rocking curve $\mathrm{FWHM}=8-12 \mathrm{arcsec}$ ) doped in the range $[\mathrm{Te}]=1 \times 10^{18}-1.6 \times$ $10^{19} \mathrm{~cm}^{-3}$. They were annealed at temperatures from the range $T=700-1200^{\circ} \mathrm{C}$ for 0.1-400 hours in quartz ampoules under vacuum and quenched in water. An annealing at a given temperature $T$ was conducted for time long enough to establish a stationary (equilibrium) state of the crystal structure at $T$, this equilibrium was then frozen by a rapid cooling [13]. We assume that the temperature of annealing is a good measure of the structural state of the sample during measurements at room temperature (RT). For X-ray studies the surface of samples was carefully chemo-mechanically polished. X-ray measurements were performed at RT in Philips Material Research Diffractometer in high resolution triple axis mode (Cu $K_{\alpha_{1}}$ radiation, 4-crystal Bartels $\mathrm{Ge}(440)$ monochromator, analyser). Hall effect was measured by van der Pauwe technique at RT.

Figure 1 presents selected results of X-ray diffraction and scattering - the maps of intensity, close to 004 reciprocal lattice (RL) point (symmetrical geometry) as a function of $q_{x}, q_{z}$ coordinates of vector $q$ in RL, where $q_{z}$ is parallel, and $q_{x}$ perpendicular to Bragg diffraction vector $h_{004}$. Constant intensity contours are traced. The characteristic surface streak [14] along the diffraction vector [004] is visible. In highly doped sample with $[\mathrm{Te}] \approx 1.6 \times 10^{19} \mathrm{~cm}^{-3}$ (Fig. $1 \mathrm{a}, \mathrm{b}$ and $\mathrm{c}$ ) the annealing at low temperature $T=800^{\circ} \mathrm{C}$ (Fig. 1c) caused about hundred times increase in intensity diffusely scattered around Bragg maximum in comparison with annealing at high temperature $T=1100^{\circ} \mathrm{C}$ (Fig. 1a). In low-doped one [Te] $\approx$ $1.3 \times 10^{18} \mathrm{~cm}^{-3}$ (Fig. 1d and e) strong DXS does not appear - similar annealing does not change low DXS intensity. Thus we conclude that strong DXS after low temperature annealing is directly related to the presence of high concentration of impurities. Moreover, an occurrence of scattering in highly doped sample after annealing below a certain temperature is a characteristic feature of the critical 

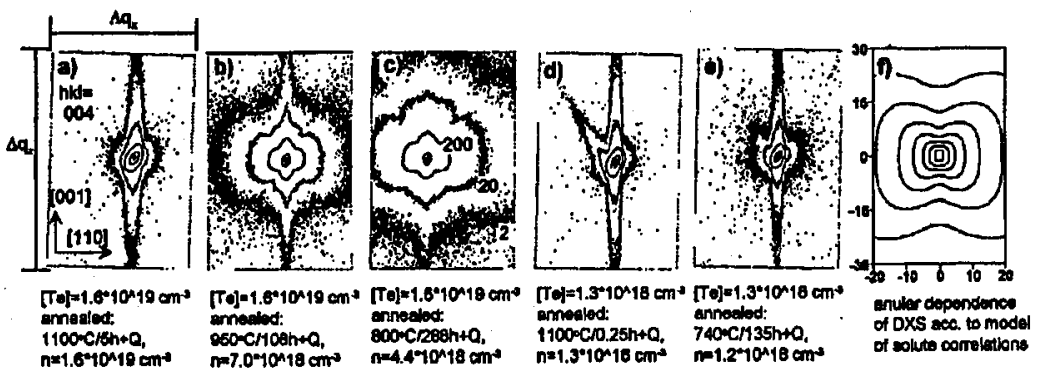

Fig. 1. Measured (a)-(e) and calculated (f) reciprocal lattice maps of X-ray intensity around 004 Bragg reflection in GaAs:Te as a function of doping level and annealing temperature. Range of wave vector $q$ coordinates: $\Delta q_{x}=37 \mu \mathrm{m}^{-1}=4.5 \times 10^{-4} \mathrm{rlu}$ $(\lambda / 2 d), \Delta q_{z}=57 \mu \mathrm{m}^{-1}=7 \times 10^{-4}$ rlu. Contours are drawn for the intensity values: $I_{\mathrm{Bragg}}=4000-7000 \mathrm{cps},(1 / 2) I_{\mathrm{Bragg}}, 200,20,2 \mathrm{cps}$. The notation, e.g. $1100^{\circ} \mathrm{C} / 5 \mathrm{~h}+\mathrm{Q}$, means an annealing at $1100^{\circ} \mathrm{C}$ for 5 hours terminated by quenching. Strong diffuse scattering (DXS) is visible around Bragg maxima in (b) and (c). The very significant $(\approx 100$ times) increase - part $(c)$ as compared with $(a)$, is seen for high Te content after low temperature annealing, and it is accompanied by free electron concentration drop. Very low DXS is visible for low doped samples - part (d) and (e), and neither DXS nor $n$ changes with annealing. High perfection of crystal (low DXS) is visible for highly doped GaAs annealed at high enough temperature (a). Calculations ( $f$ ) of angular distribution of DXS according to the model of impurity correlations (no surface streak was modelled) show close similarity to angular dependence from (b) and (c).

scattering. This strongly indicates that very highly doped GaAs:Te solid solution undergoes a kind of structural transition.

The results of DXS were in a very good coincidence with changes of the free electron concentration $n-$ an increase in DXS was accompanied by a drop of $n$. Both effects were reversible, DXS decreased and $n$ increased after a second annealing at sufficiently high temperature. In low doped crystals neither DXS nor $n$ were changed by the annealing. Thus both results, reversible diffuse $\mathrm{X}$-ray scattering and drop/rise of the free electron concentration, come from mutually related behaviours of impurities.

In Fig. $1 b$ and $c$ one can see that the dominating intensity of arising DXS lies in the direction perpendicular to the scattering vector along [001] and isointensity contours have an oval shape (very well visible for earlier stages of a transition Fig. 1b). In above-mentioned scattering description ([8], Ch. 3 of Ref. [11]) such angular dependence is a very characteristic mark of lattice deformations related with solute atom-atom spatial correlations fluctuating in a solid solution. In Fig. If the calculations of isointensity lines in RL according to the described model of fluctuating correlations are presented for a simplified lattice (fcc instead of zinc blende) and correlations at first neighbour sites. As concluded by Krivoglaz [8, 11], the oval shape of isointensity lines does not depend on microscopic details of solute atom-atom spatial correlations (vector $\rho=r_{i}-r_{j}$ ). Therefore such scattering does not allow to determine these details, although it allows to infer on correlations. 
In conclusion, the three reported features of measured DXS in the very highly doped GaAs:Te solid solution, namely its relation with doping level, its critical type dependence on annealing temperature and good description of its intensity in reciprocal space with the referred model allow us to state that one deals with critical $\mathrm{X}$-ray scattering due to fluctuations of impurity spatial correlations in GaAs:Te solid solution. It means that the spatial correlations of impurities occur in a crystal, inhomogeneously in a crystal volume, i.e. regions of low and high value of the correlation function are formed at structural equilibrium. An arising of correlations in initially random solid solution is a kind of structural phase transition.

The above-mentioned decrease in the free electron concentration occurring simultaneously with an arising of impurity correlations inferred from X-ray studies strongly suggests a certain form of impurity bonding. Recently, an existence of diatomic centres of bonded impurities (with equivalent both sites) in very highly doped GaAs: $\mathrm{C}$ in a similar situation (the changes caused by an annealing) has been proved by Raman spectroscopy in annealed crystals doped with $\mathrm{C}$ isotopes [15].

T.S. thanks Prof. M. Grynberg and Prof. J.A. Kozubowski for many discussions and support. This work was partially supported by the Committee for Scientific Research (grant No. 3P407-031-07). MRD diffractometer was granted by the Foundation for Polish Science.

\section{References}

[1] M.E. Fisher, Rep. Prog. Phys. 30, 615 (1967).

[2] P. Heller, Rep. Prog. Phys. 30, 731 (1967).

[3] R.J. Elliott, W. Marshall, Rev. Mod. Phys. 30, 75 (1958).

[4] G.A. Baker Jr., Quantitative Theory of Critical Phenomena, Academic Press, Boston 1990.

[5] J. Als-Nielsen, O.W. Dietrich, Phys. Rev. 153, 706, 711, 717 (1967).

[6] B.E. Warren, X-ray Diffraction, Dover Publ., New York 1969, 1990.

[7] J.M. Cowley, J. Appl. Phys. 21, 24 (1950).

[8] M.A. Krivoglaz, Fiz. Met. Metalloved. 8, 648 (1959).

[9] A.A. Maradudin, E.W. Montroll, G.H. Weiss, I.P. Ipatova, in: Solid State Physics, Eds. H. Ehrenreich, F. Seitz, D. Turnbull, Academic Press, New York 1971.

[10] H. Ekstein, Phys. Rev. 68, 121 (1945).

[11] M.A. Krivoglaz, Diffuse Scattering of $X$-rays and Neutrons by Fluctuations, Springer-Verlag, Berlin 1996 (transl. from Russian ed. 1984).

[12] C.S. Fuller, K.B. Wolfstirn, J. Appl. Phys. 34, 2287 (1963).

[13] T. Słupiński, E. Zielińska-Rohozińska, T. Harasimowicz, Acta Phys. Pol. A 90, 1080 (1996).

[14] S.R. Andrews, R.A. Cowley, J. Phys. C 18, 6427 (1985).

[15] J. Wagner, R.C. Newman, B.R. Davidson, S.P. Westwater, T.J. Bullough, T.B. Joyce, C.D. Latham, R. Jones, S. Öberg, Phys. Rev. Lett. 78, 74 (1997). 